"The final publication is available at Springer via http://dx.doi.org/10.1023/B:JOIH.0000030227.41379.13". 


\title{
Predictors of Clinical Breast Examination aMong SOUTH ASIAN IMMIGRANT WOMEN
}

\author{
Farah Ahmad ${ }^{1,2}$ and Donna E. Stewart ${ }^{1,2}$ \\ ${ }^{1}$ Women's Health Program, University Health Network, Toronto, Canada \\ ${ }^{2}$ Faculty of Medicine, University of Toronto, Toronto, Canada
}

Running Title: SA Women and Clinical Breast Exam

Correspondence: $\quad$ Farah Ahmad, MBBS, MPH, PhD (Candidate)

University Health Network

657 University Avenue, ML 2-010A

Toronto, Ontario, M5G 2N2, Canada

Telephone: (416) 340-4800 ext. 6987

Fax: (416) 340-4185

E-mail: farah.ahmad@uhn.on.ca 


\begin{abstract}
Objective: To determine predictors of clinical breast examination (CBE) among South Asian immigrant women residing in Toronto, Canada.
\end{abstract}

Methods: A cross-sectional self-administered survey with women patients visiting family physician group practices.

Results: Fifty-four women participated in the study (response rate 77\%). Twenty women (38.5\%) "ever had" CBE. Compared to women who never had CBE, women who had CBE were statistically older, had lived more years in Canada, had better knowledge of breast cancer, had lower perceived barriers to $\mathrm{CBE}$, and were more likely to have ever had a periodic health exam. No significant differences were found between the two groups for education, employment, English language abilities, perceived health, and perceived benefits of CBE. A direct logistic regression with five predictor variables, significant at a univariate level, was statistically reliable, $X^{2}(5, \mathrm{n}=51)=34.7, \mathrm{p}<0.001$ and explained $67 \%$ of the variance in the CBE status. Age and perceived barriers to $\mathrm{CBE}$ remained significant over and above other predictor variables. The odds of 'ever had' CBE increased with age and decreased with more perceived barriers.

Conclusion: The study highlights the need for education interventions on breast cancer and screening among SA recent immigrant women.

Keywords: clinical breast examination, South Asian, predictors, Health Belief Model 


\section{Predictors of Clinical Breast Examination AMong SoUth Asian IMMigrant Women}

\section{INTRODUCTION}

Breast cancer is the most commonly diagnosed cancer among North American women. Early cancer diagnosis leads to a more favorable prognosis and survival rates which makes breast self-examination (BSE), clinical breast examination (CBE) and screening mammography important health behaviors for women (1). However, breast cancer screening programs are underutilized by ethnic women in North America $(2,3)$. This gap is an especially salient concern in Canada due to its increasing population diversity. Since globalization of its immigration policy in the 1970 's, most of recent immigrant women are visible minorities and South Asians ${ }^{1}$ are the second largest but fastest growing group $(4,5)$. Although South Asian (SA) migrants to North America have moved from low- to high-risk countries with respect to breast cancer, evidence suggests convergence of their breast cancer age-standardized mortality rates with the host population (6). Saphir cites similar findings and notes a "false sense of security" among Asian Americans that they are healthier than their White counterparts (7).

Likewise, recent qualitative studies with SA immigrant women reveal their low perceived risk of breast cancer but high fear, fatalistic beliefs, belief in inevitability of suffering due to fate (karma), and embarrassment in undergoing sensitive physical examinations $(8,9)$. Other qualitative studies report limited breast health knowledge among SA immigrant women (10). Collectively, these barriers, misperceptions and limited knowledge are likely to hinder SA women from performing or seeking breast examinations. For instance, a pilot study by Choudhry in 1998 suggested low uptake of breast cancer screening among SA immigrant women residing

\footnotetext{
${ }^{1}$ Immigrants originating from India, Pakistan, Sri Lanka, and Bangladesh are categorised as South Asian in Canadian Population Census data.
} 
in Toronto (10). The data, gathered by open-ended questions, identified that only $12 \%$ of women practiced BSE and $49 \%$ had at least one CBE. In addition, coding of interviews by constructs of the Health Belief Model followed by an analysis of variance found "cues-to-action" as the only significant variable for women's breast health practices. Addressing women's perceived barriers and information gaps is a pre-requisite for regular uptake of breast screening procedures even when performed by health care providers. However, existing scholarly knowledge on the breast cancer screening behavior of SA immigrant women is scattered and needs comprehensive investigation.

To advance understanding, our paper presents results of a pilot data gathered for our ongoing health promotion project that uses SA ethnic newspapers to improve awareness and uptake of breast cancer screening, especially CBE and mammogram. This focus was established based on Canadian guidelines that recommend CBE annually, by a physician or specially trained nurse, for all adult women and mammograms every 2 years for women aged 50-74 (11). Consistent with the focus of our community oriented health promotion project, this pilot study investigates predictors of $\mathrm{CBE}$ from women's perspectives.

The study utilized the conceptual framework of Health Belief Model (HBM) introduced by Hochbaum in the early 1950s (12), which for the last five decades has been one of the most widely used explanatory and predictive model in health behavior research and interventions (13). Our study included the core constructs of the HBM i.e. perceived susceptibility; perceived seriousness; perceived benefits; perceived barriers; and cues-to-action. Studies have demonstrated that the constructs of perceived barriers and benefits are the most significant predictors of breast cancer screening behavior (14). Theoretically perceived susceptibility and seriousness are primarily determined by the individual's knowledge of the disease and personal risk; hence, knowledge was measured directly rather than by perceived seriousness and susceptibility. Based 
on a literature review and guided by the HBM, our pilot study hypothesized that the likelihood of 'ever having had a CBE' would be predicted by a) younger age; b) higher level of acculturation; c) higher knowledge about breast cancer; d) having heard of CBE (cues-to-action); e) lower perceived barriers to $\mathrm{CBE}$; and f) higher perceived benefits of CBE.

\section{METHODS}

Study Design and participants

The study used a cross-sectional survey design with self-administered questionnaires completed at site of contact. A convenience sample size of approximately 50 women was selected for this study. The survey instrument was translated in Hindi and Urdu by bilingual research assistants. The accuracy of the translated versions was checked by two community members and one of the authors (F.A.) fluent in the respective languages. The translated versions were pilot tested for clarity and comprehension by two separate focus groups with Urdu and Hindi speaking SA immigrant women. Ethics approval was obtained from the institution's research ethics board.

Eligibility criteria included being a South Asian immigrant woman at least 18 years of age who could speak and read Hindi or Urdu. Using the online database of the College of Physicians and Surgeons of Ontario, 17 family physicians who spoke the language of the targeted group were identified in the Greater Toronto Area. The study details and request for collaboration were sent to six physician offices that were selected on the basis of their group practice setting and their report of a sizeable Hindi or Urdu speaking clientele. This selection was intentional to control for health-care-access and English language proficiency as barriers to breast cancer screening. Three group practice clinics located in middle-income neighborhoods 
with a higher proportion of SA residents collaborated for the study. The study subjects were recruited during work days for 3 consecutive weeks, 1 week at each clinic. All eligible women visiting the clinic during the study period were invited to participate in the study. The study was introduced to the potential participants as a survey with South Asian immigrant women on their knowledge and attitudes about breast cancer screening. Willing participants provided written consent prior to completing the questionnaire individually in a private corner of the waiting area that was specifically allocated for this purpose.

\section{Measurement and Statistical analyses}

The survey instrument gathered information on sociodemographic characteristics; breast cancer related knowledge; $\mathrm{CBE}$ screening status; ever heard of $\mathrm{CBE}$; and perceived barriers and benefits to CBE. The sociodemographic data was collected on age, marital status, children, years lived in Canada (proxy for acculturation), education, employment status, English speaking abilities, periodic health examination status, and perceived health. Women rated their English speaking abilities and perceived health on a scale of 1-5 (poor to excellent). The study used the number of years lived in Canada as a proxy of acculturation as none of the existing scales were translated and validated for the targeted group.

Breast cancer related knowledge was assessed by 12 items on breast cancer risk, causes, symptoms, and screening methods (see Appendix A). The items were derived by a literature review (8-10) and developed according to Canadian screening guidelines. For instance, these questions asked about women's lifetime chance of getting breast cancer, risk of breast cancer in various age groups, recommended screening methods for women 50 years of age and over, and recommended time intervals for screening. For each knowledge item, the study subjects selected one statement that appeared 'true' to them from a provided set of statements. The correct answers 
were coded as 1 , and false or do not know as 0 . Subsequent results are based on mean knowledge derived across 12 items with a maximum possible score of 1 and a minimum possible score of 0 .

The outcome variable CBE screening status was assessed by 'ever had' CBE (yes/no). A brief definition of $\mathrm{CBE}$ screening was included in the questionnaire prior to $\mathrm{CBE}$ specific questions. Women who had CBE were asked about the time of their last examination (less than one year, between 1-3 years, more than 3 years). The HBM construct of cues-to-action was operationalized by one item that asked women whether they have ever heard of this examination.

Perceived barriers ( 8 items) and perceived benefits of CBE ( 5 items) were derived from published studies $(14,15)$. For each of these statements, respondents selected one option from strongly disagree, disagree, agree, and strongly agree on a scale of 1-4, respectively. A list of these items is included in Appendix A. The items on perceived benefits of CBE included: feeling good about oneself; benefit to the family; less worry; early detection of lumps; and decrease chance of dying from breast cancer. The items on perceived barriers to CBE included: limited knowledge about the procedure, who performs it and where; other things being more important; more worry; embarrassment; time constraints; and discomfort. The constructs of perceived barriers to $\mathrm{CBE}$ and perceived benefits of $\mathrm{CBE}$ were operationalized by deriving two variables of means across items. For these derived variables, a value of 2 or less refers to some level of disagreement, and values more than 2 refer to some level of agreement. Internal consistencies of perceived benefits and barrier scales were demonstrated by Cronbach's alpha of 0.63 and 0.81 , respectively.

Statistical analyses included descriptive statistics with a correction of outliers and missing values which were replaced by group specific means for continuous variables. Univariate analyses comprised of the Chi-square or Fisher Exact test, Student $t$-test, point- 
biserial correlation. The Student $t$-test was applied only if variables were normally distributed and point-biserial correlation only for skewed variables. At a multivariate level, direct logistic regression compared the constant-only model with the full model that had all the predictors (16). Due to our restricted sample size, variables that had a significant association to the CBE status at the univariate level were entered in the logistic regression analysis. The probability of making a type-I error was set at 0.05 with a two-sided significance. Income was not included in the analyses as $27 \%$ of the data were missing. The data were analyzed using a Statistical Package for the Social Sciences (SPSS) version 10.1.

\section{RESULTS}

Seventy eligible women were invited to participate and 54 women completed the surveys (response rate 77\%). Two outliers for 'number of years lived in Canada' were deleted after unsuccessful transformation. Hence, the results are based on 52 subjects.

Overall results

On average, women were 36.7 years old ( $\mathrm{SD} \pm 9.1$, range 25 to 60 ) and had lived approximately 6 years in Canada ( $\mathrm{SD} \pm 6.7$, range 1 to 16 ). All women were married ( $92 \%$ currently married) and $86.5 \%$ had children. Seventy-one percent of the women had some or completed university education and $18.1 \%$ were employed. English speaking ability was rated on a scale of 1 to 5 as 'good' (mean $=2.7, \mathrm{SD} \pm 1.4$ ). Women perceived their health as 'good' on a scale of 1 to 5 (mean $=3, \mathrm{SD} \pm 1.0$ ). Forty-one percent of the women had at least one periodic health exam.

Although $83 \%$ of the women had heard of CBE, only $38.5 \%$ of the women 'ever had' $\operatorname{CBE}(\mathrm{n}=20)$. Among women who had CBE, $57.9 \%$ had the exam within the last year, $31.6 \%$ in 1 
to 3 years and $10.5 \%$ in more than 3 years. Approximately, $90 \%$ of women agreed with perceived benefits of $\mathrm{CBE}$. At the same time, $81 \%$ agreed with perceived barriers to $\mathrm{CBE}$.

Overall knowledge mean score across 12 items was less than average (mean $=0.3, \mathrm{SD}$ \pm 0.2 , possible minimum and maximum score, 0 to 1 ); $17 \%$ of women were unable to provide any correct answers and $73 \%$ had correct answers to less than $50 \%$ of questions. A detailed analysis of responses to knowledge items was executed. Few women accurately knew that mammogram for women 50 and over is recommended every two years (7.4\%); lifetime chance of getting breast cancer is 1 in $9(9.3 \%)$; breast screening methods for women 50 and over include CBE, BSE and mammogram (20.4\%); association of pain with early breast cancer is unusual (24\%); and radiation during mammography is not a serious risk (26\%). Additionally, only $44 \%$ of women knew that mammogram is universal health service in Canada and cost free to individuals.

\section{Group Comparison}

In this paper, the group of women who 'ever had' CBE is referred as G-1 and the group who 'never had' CBE as G-0. Group comparison between G-1 and G-0 for the aforementioned variables revealed statistically significant differences for age, number-of-years lived in Canada, periodic health exam, knowledge, and perceived barriers to CBE (see Table I). The G-1 women were older in age and had lived more years in Canada compared to G-0 women. More G-1 women ever had a routine physical checkup compared to G-0 women (60\% and $29 \%$, respectively). Women in G-1 had a statistically higher mean knowledge score and perceived lower barriers to CBE compared to G-0 women.

Further item analysis of the perceived barriers reveal that the top three significant barriers among G-0 women compared to G-1 were 'do not know where to go' (mean: 2.8 vs. 2.1); 'do not understand how $\mathrm{CBE}$ is done' (mean: 2.8 vs. 1.9); and 'do not know who to ask' (mean: 2.8 vs. 
1.8). Other significant barriers were 'CBE every year will make me worry'; 'CBE takes too much time'; and 'CBE is uncomfortable'. No significant differences were found between the two groups for marital status, education, employment, English language abilities, perceived health, ever heard of CBE, and perceived benefits of CBE.

\section{Likelihood of 'ever had' CBE}

Direct logistic regression analysis was executed with CBE status as the outcome variable and five predictor variables: age, years lived in Canada, periodic health exam, knowledge mean score, and perceived barriers to CBE. A test of the full model against the constant-only model was statistically reliable, $X^{2}(5, \mathrm{n}=51)=34.7, \mathrm{p}<0.001$, indicating that the predictors, as a set, reliably distinguished between women who ever had CBE and women who never had CBE. It explained 0.67 Nagelkerke R Square variance in the CBE status. The prediction success was $87.1 \%$ for women who never had $\mathrm{CBE}$ and $65 \%$ for women who ever had $\mathrm{CBE}$, for an overall success rate of $78.4 \%$ for accurate classification of women's CBE status.

The importance of the predictors was assessed by Wald criterion (Table II). According to Wald criterion predictor age and perceived barriers to CBE remained significant over and above other predictor variables. The odds of 'ever had' CBE decreased by $96 \%$ with a one unit increase in the perceived barriers. The odds of 'ever had' CBE increased by $17 \%$ by a one year increase in age. Also, the direction of prediction for age was not the same as hypothesized i.e. being younger would increase the likelihood of having CBE. The study results indicate that higher age increased the likelihood of having a CBE in this immigrant population.

For the logistic regression analysis, the data set had an adequate ratio of cases-tovariables i.e. 10 cases per predictor (17) and did not violate the assumption of linearity in the logit. Also, multicollinearity was absent in the predictor variables. 
Table I: Univariate Predictors of Clinical-Breast-Examination

\begin{tabular}{|c|c|c|c|c|c|}
\hline \multirow[t]{3}{*}{ Variable } & \multicolumn{2}{|c|}{ Descriptive } & \multicolumn{3}{|c|}{ Significance test } \\
\hline & Group-1 $^{a}$ & Group-0 $^{b}$ & $T$-test & Point-biserial & $X^{2}$ \\
\hline & $(n=20)$ & $(n=32)$ & & & \\
\hline Age, mean ** & $42.2(\mathrm{SD} 9.2)$ & $33.3(\mathrm{SD} 7.3)$ & & 0.48 & \\
\hline Years lived in Canada, mean ** & $9.6(\mathrm{SD} 9.1)$ & 3.5 (SD 2.8) & & 0.45 & \\
\hline Ever had routine physical, $\% *$ & $60 \%$ & $29 \%$ & & & 4.8 \\
\hline Knowledge-score ${ }^{\mathrm{c}}$, mean $* *$ & $0.4(\mathrm{SD} 0.3)$ & $0.2(\mathrm{SD} 0.2)$ & 2.9 & & \\
\hline CBE-barriers, mean ${ }^{\mathrm{d} * *}$ & $1.9(\mathrm{SD} 0.5)$ & $2.6(\mathrm{SD} 0.5)$ & 4.3 & & \\
\hline \multicolumn{6}{|l|}{ * significant at 0.05} \\
\hline \multicolumn{6}{|l|}{$* *$ significant at 0.01} \\
\hline \multicolumn{6}{|l|}{ a women who ever had CBE } \\
\hline \multicolumn{6}{|l|}{ b women who never had CBE } \\
\hline c maximum possible score 1 anc & minimum possi & ble score 0 & & & \\
\hline
\end{tabular}




\section{Table II: Logistic Regression Analysis: Predictors of Clinical Breast Examination}

\begin{tabular}{|c|c|c|c|c|c|c|}
\hline \multirow[t]{2}{*}{ Variables } & \multirow[t]{2}{*}{$B^{a}$} & \multirow[t]{2}{*}{ Wald Test } & \multirow[t]{2}{*}{ Significance } & \multirow[t]{2}{*}{ Odds Ratio } & \multicolumn{2}{|c|}{$\begin{array}{l}95 \% C^{b} \\
\text { Odds ratio }\end{array}$} \\
\hline & & & & & upper & lower \\
\hline Age & 0.16 & 5.4 & 0.02 & 1.17 & 1.03 & 1.34 \\
\hline Years lived in Canada & 0.20 & 2.0 & 0.14 & 1.22 & 0.93 & 1.61 \\
\hline Routine physical checkup (yes=1) & 0.48 & 0.4 & 0.65 & 1.62 & 0.19 & 13.3 \\
\hline Knowledge-score & 1.35 & 0.4 & 0.53 & 3.85 & 0.06 & 248 \\
\hline CBE-barriers & -3.29 & 5.4 & 0.02 & 0.04 & 0.00 & 0.59 \\
\hline (Constant) & -1.69 & 0.1 & & & & \\
\hline
\end{tabular}

\footnotetext{
${ }^{a}$ B: coefficient estimated for the model

${ }^{\mathrm{b}} \mathrm{CI}$ : confidence interval
}

\section{Discussion}

The study findings advance understanding of predictors of clinical breast examination among South Asian immigrant women and the existing gaps that need to be targeted by future interventions. Despite the fact that all participant women had a regular source of healthcare, no language communication barrier with physicians (due to our recruitment strategy), and twothirds reported having heard of breast screening methods (i.e. high cue-to-action), only $38.5 \%$ ever had CBE. This finding emphasizes the need to tailor the information and not just convey the message. Encouragingly, most of those who had CBE followed the recommended frequencies. In this study, the likelihood of ever having had a CBE increased with age (being older) and decreased with higher perceived barriers over and above predictors of years lived in Canada, 
knowledge, and periodic health examination status. The full model had an overall success rate of $78 \%$ for accurately classifying women's CBE status thereby it is likely to contribute towards the development of effective health promotion programs for this population.

It is acknowledged that our study results are limited in establishing a strict cause and effect relationship due to our cross-sectional design; future longitudinal studies would be beneficial. Despite the restricted generalizability due to the relatively small sample size of this pilot study, the results are anticipated to benefit programs serving recent SA immigrant women, particularly Urdu and Hindi speaking, residing in urban areas. The interpretation of results also requires consideration to differences in the way health care services are provided across countries, as the study was conducted in Canada which has a universal health care system. As the study findings are limited to Urdu or Hindi speaking SA women's perspectives recruited from family practice clinics in middle-income neighbourhoods, future studies with larger samples should include diverse socioeconomic and language subgroups within SA category. Future work also needs to investigate physicians' barriers in conducting CBE among SA women.

The study demonstrates a significant association between relevant knowledge and breast cancer screening status of SA immigrant women. Although a causal relationship can not be established, social cognitive theory states that adequate knowledge is a prerequisite to the performance of any particular health behavior (18). This finding is also in agreement with other studies of South Asian women regarding cervical cancer screening behavior (19).

The knowledge gap about breast cancer found by this study is of concerning. Only a quarter of the participants gave correct answers to at least half the questions in the knowledge scale, despite the fact that almost two-third had at least some university education. A possible explanation may lie in the fact that SA immigrants originated in countries where acute and 
infectious health conditions hold priority over chronic diseases like cancers in health resource allocation. These systemic differences between regions of South Asia and North America may account for low knowledge about breast cancer in this population. This is reflected in our study results; for instance, within five knowledge items that were answered accurately by less than one-fourth of the participants, three items were related to breast screening intervals and procedures. There is a strong need to educate recent SA immigrant women about breast cancer and recommended screening methods. However, human behavior is complex and involves many facets in addition to adequate knowledge.

The study participants perceived the benefits of CBE in similar ways regardless of whether they had experienced it. However, women who never had CBE perceived statistically higher barriers to $\mathrm{CBE}$ compared to women who ever had CBE. This finding is in congruence with other studies among women of the majority culture and some ethnic subpopulations $(14,15)$ but, to our best knowledge, this study is the first to empirically demonstrate this in SA immigrant women. Among women who never had a CBE, the top three barriers were 'do not know where to go'; 'do not understand how CBE is done'; and 'do not know who to ask'. Other significant barriers included worries stemming from having CBE every year, too much time required to conduct $\mathrm{CBE}$, and discomfort. These findings support the need to transfer health knowledge into health behaviors through tailored health promotion messages that emphasize issues specific to the targeted community.

In addition to tailored education at the community level to reach those outside the medical care setting, healthcare providers influence patients' breast cancer screening behavior (20). In our study, SA women who ever had a periodic health examination were more likely to have a CBE but only $41 \%$ of all the participants ever had a routine physical exam. One possible 
explanation may lie in participants' limited preventative health orientation. For instance, other studies report that SA immigrant women may view healthcare visits necessary only when symptoms exist (8). Other studies suggest that SA women's unshared work burden, embedded in patriarchal social system and traditional gender roles, leads to limited time for seeking preventative health services $(21,22)$. Hence, comprehension of the concept of screening may be difficult for some recent SA immigrants. In this study, women who never had a periodic health exam were more recent migrants than women who had the exam thereby underscoring the importance of promoting routine physical checkups in recent migrants. Healthcare providers need to be vigilant about low rates of periodic health examination in the studied group.

Several large scale North American survey studies examining the relationship between demographic variables and participation in screening report that higher education and income increases women's likelihood of having breast or cervical cancer screening $(23,24)$ while increasing age decreases the likelihood of CBE (25). Our study finds a significant association between age and women's CBE screening, at both univariate and multivariate level, but in the reverse direction i.e. likelihood of ever had CBE increased with an increase in women's age. Future studies with larger samples should investigate this finding further.

Behavior often changes as a result of acculturation following migration. In this study, women's CBE status was associated with the number of years spent in Canada, a proxy measure of acculturation. Women were more likely to have CBE if they had lived more years in Canada demonstrating the influence of environment on behavior. Hoare et al reports a similar influence on the cancer screening behavior of Asian women living in UK (26). Other studies show that after living ten years in the adopted country the cancer screening behaviors of immigrant women 
converge with the host population (27) illustrating the need of a proactive healthcare system to address the variability of need within a group.

In conclusion, our study findings indicate a need for community education among SA recent immigrant women on early detection of breast cancer through screening. Future effective health promotion initiatives need to address women's perceived barriers to motivate their uptake of breast cancer screening. It also seems that healthcare providers need to enhance their efforts towards promotion of preventative health examinations, including CBE, in the studied group. 


\section{Appendix A}

\section{$\underline{\text { Knowledge items }}$}

1. On average how many women in Canada will get breast cancer sometime during their lives?

2. Who do you think is more likely to get breast cancer in following age groups?

3. Who do you think is more likely to get breast cancer (close relative with breast cancer)?

4. What is the recommended interval for breast examination (BSE) by yourself?

5. Which of the following interval is recommended for women 50 years of age and over to have a mammography or breast x-ray?

6. Which of the following method(s) is/are recommended for women 50 years of age and over?

7. Can bumping or bruising cause breast cancer?

8. Can breast cancer spread from one woman to another woman?

9. Is pain usually associated with early breast cancer?

10. Can radiation exposure during a mammogram be a serious risk to women?

11. Should a non-pregnant woman see her physician if she has discharge from her nipple(s)?

12. Do women have to pay for a mammogram in Canada?

\section{Perceived Benefits of $C B E$}

1. After I will get a clinical breast examination, I will feel good about myself.

2. After I will get a clinical breast examination and nothing is found, I will not worry as much about cancer. 
3. Having a clinical breast examination will decrease my chances of dying from breast cancer.

4. Having a clinical breast examination will help me find breast lumps early.

5. Having a clinical breast examination will benefit my family.

\section{Perceived Barriers to CBE}

1. I do not know where to go for a clinical breast examination.

2. I do not know who to ask about a clinical breast examination.

3. I do not understand how a clinical breast examination is done.

4. I have other problems more important than getting a clinical breast exam.

5. Having a clinical breast examination every year will make me worry about breast cancer.

6. Having a clinical breast examination is too embarrassing.

7. Having a clinical breast examination takes too much time.

8. Having a clinical breast examination is uncomfortable 


\section{ACKNOWLEDGMENTS}

The study results are based on preliminary data collected for a health promotion project funded by the Canadian Breast Cancer Foundation. We acknowledge contributions by Jill Cameron and Ilene Hyman who were members of the research team. Also, we thank Ms. Reena Vanza and Amena Syed for their assistance in the data collection. 


\section{REFERENCES}

(1) Gaudette LA, Silberberger C, Altmayer CA, Gap RN: Trends in breast cancer incidence and mortality. Health Rep 1996; 8(2):29-37.

(2) Maxwell CJ, Kozak JF, Desjardins-Denault SD, Parboosingh J: Factors important in promoting mammography screening among Canadian women. Can J Public Health 1997; $88(5): 346-350$.

(3) Maxwell CJ, Bancej CM, Snider J: Predictors of mammography use among Canadian women aged 50-69: findings from the 1996/97 National Population Health Survey. CMAJ 2001; 164(3):329-334.

(4) Statistics Canada: Women in Canada 2000: a gender-based statistical report. 89-503XPE. 2000. Ottawa, Housing, Family and Social Statistics Division.

(5) Statistics Canada: Immigrant population by place of birth and period of immigration, 1996 Census, Canada. http://www.statcan.ca/english/Pgdb/People/Population/demo25a.htm.

(6) Kliewer EV, Smith KR: Breast cancer mortality among immigrants in Australia and Canada. J Natl Cancer Inst 1995; 87(15):1154-1161.

(7) Saphir A: Asian Americans and cancer: discarding the myth of the "model minority". J Natl Cancer Inst 1997; 89(21):1572-1574. 
(8) Bottorff JL, Johnson JL, Bhagat R, Grewal S, Balneaves LG, Clarke H, Hilton BA: Beliefs related to breast health practices: the perceptions of South Asian women living in Canada. Soc Sci \& Med 1998; 47(12):2075-2085.

(9) Johnson JL, Bottorff JL, Balneaves LG, Grewal S, Bhagat R, Hilton BA, Clarke H: South Asian women's views on the causes of breast cancer: images and explanations . Pt Edu Couns 1999; 37(3):197-306.

(10) Choudhry UK, Srivastava R, Fitch MI: Breast cancer detection practices of south Asian women: knowledge, attitudes, and beliefs. Oncol Nurs Forum 1998; 25(10):1693-1701.

(11) Ontario Breast Screening Program. Cancer Care Ontario. http://www.cancercare.on.ca/obsp.

(12) Hochbaum GM: Public participation in medical screening programs: a sociopsychological study. Public Health Service 1958; 572.

(13) Strecher VJ, Rosenstock IM: The Health Belief Model: In: Glanz K, Lewis FM, Rimer BK, ed. Health Behavior and Health Education. San-Francisco, CA: Joseey-Bass Inc.; 1997: 41-59.

(14) Champion VL: Revised susceptibility, benefits, and barriers scale for mammography screening. Res Nurs \& Health 1999; 22(4):341-348.

(15) Champion VL, Scott C: Reliability and validity of breast cancer screening belief scales in African American women. Nurs Res 1997; 46(6):331-7. 
(16) Tabachnick BG, Fidell LS: Using Multivariate Statistics. 3 ed. Boston, MA: Allyn and Bacon; 2001.

(17) Norman GR, Streiner DL: Logistic regression: In: Norman GR, Streiner DL, ed. Biostatistics: The Bare Essentials. Hamilton, Canada: B.C. Decker Inc.; 2000:139-144.

(18) Bandura A, Wilson GT, Kunkel J, Neale JM, Liebert RM: Social Learning Theory. Englewood Cliffs, NJ: Prentice-Hall; 1977.

(19) Gupta A, Kumar A, Stewart DE: Cervical cancer screening among South Asian women in Canada: the role of education and acculturation. Health Care for Women International $2002 ; 23(2): 123-134$.

(20) Ahmad F, Stewart DE, Cameron JI, Hyman I: Rural physicians' perspectives on cervical and breast cancer screening: a gender-based analysis. J Women Health Gen-B 2001; 10(2):201-208.

(21) George U, Ramkissoon S: Race, gender and class: interlocking oppressions in the lives of South Asian women in Canada. AFFLIA: Journal of Women and Social Work 1998; 13:102-119.

(22) Naidoo JC, Davis JC: Canadian South Asian women in transition; a dualistic view of life. Journal of Comparative Family Studies 1988; XIX(2):311-327.

(23) Goel V: Factors associated with cervical cancer screening: results from the Ontario Health Survey. Can J Public Health 1994; 85(2):125-127.

(24) Gentleman JF, Lee J: Who doesn't get a mammogram? Health Rep 1997; 9(1):19-34. 
(25) Katz SJ, Hofer TP: Socioeconomic disparities in preventive care persist despite universal coverage. Breast and cervical cancer screening in Ontario and the United States. JAMA 1994; 272(7):530-534.

(26) Hoare T, Thomas C, Biggs A, Booth M, Bradley S, Friedman E: Can the uptake of breast screening by Asian women be increased? A randomized controlled trial of a link worker intervention. J Public Health Med 1994; 16(2):179-185.

(27) Mercer SL, Goel V: Factors associated with the use of mammography: the Ontario Health Survey. Cancer Prev Control 1997; 1(2):144-151. 